\title{
Do Recognitive Factors and General Balance of the Elderly Predict Recurrent Falls? -A Prospective Study-
}

\author{
Yuri Inoue, $\mathrm{PT}^{1)}$, Kazuya Sakatomo, $\mathrm{PT}^{2)}$, Toshiyuki Sako, $\mathrm{PT}^{3)}$, \\ Yoshihiro Takeuchi, $\mathrm{CW}^{4}$, Ryoma Nakagoshi, $\mathrm{PT}^{5)}$, Kunihiro Sumihito, $\mathrm{PT}^{6}$, \\ Kazuki Maetani, $\mathrm{OT}^{7}$ ), Kazuhiro Matsuda, $\mathrm{PT}^{8)}$, Seitci Takemasa, PT, PhD ${ }^{1)}$, \\ Masahito Murakami, PT, $\mathrm{PhD}^{1)}$ \\ 1) Faculty of Rehabilitation Science, Kobe International University: 9-1-6 Koyocho, Higashinada, Kobe \\ City, Hyogo 658-0032, Japan. TEL: +81 78-940-4118,E-mail: inoue@kobe-kiu.ac.jp \\ 2) Department of Day-care, Nakabayashi Hospital \\ 3) Day-care center, Yagi Hospital \\ 4) Department of Day-care, Kawakami Orthopedic Clinic \\ 5) Department of Rehabilitation, Health Care Service Facility for the Aged, Elder Village \\ 6) Department of Rehabilitation, Higashiura Heisei Hospital \\ 7) Department of Rehabilitation, Health Care Service Facility for the Aged Tsuna Hakujuen \\ 8) Department of Rehabilitation, Nakayama Clinic
}

\begin{abstract}
Purpose] The purpose of this study was prospectively investigated whether recognitive factors and general balance of the elderly influence recurrent falls. [Subjects] The participants were 85 elderly, cared for at day care centers, who were followed for one year. [Methods] At baseline, we evaluated fall-related self-efficacy using the Modified Falls Efficacy Scale (MFES), and measured the gap between the actual reach distance and the estimated reach distance (GAE) as a recognitive factor. We also evaluated balance using the Timed Up and Go Test (TUG). For the follow-up, we interviewed the participants about the number of times they had fallen in the past year. We assigned whether the participants had fallen more than twice at follow-up to a dependent variable, and with MFES, GAE and TUG at baseline as independent variables, we performed a multiple logistic regression analysis to explore the factors which were related to recurrent falls. [Results] Fourteen participants had fallen more than twice, and it was found that GAE was related to recurrent falls. [Conclusion] GAE could be used as a factor for predicting the risk of recurrent falls.

Key words: Perception of postural limits, Fall-related self-efficacy, Recurrent falls
\end{abstract}

(This article was submitted Feb. 29, 2012, and was accepted Mar. 28, 2012)

\section{INTRODUCTION}

The causes of falls in the elderly can be classified as internal factors and external factors. In addition, internal factors can be classified as physical, sensory, and recognitive factors ${ }^{1}$. Recently, recognitive factors, including fear of falling and inadequate perception of postural limits, have been widely investigated.

Fear of falling is defined as a permanent fear of doing an activity in which movement or a change in position is required. When elderly people have an intense fear of falling, they tend to limit some of their activities, in spite of having the necessary physical abilities to do them. This may lead to a decline in their activities of daily living (ADLs), leading to an increase in the risk of falling ${ }^{2)}$.

Tinetti ${ }^{2}$ designed the Falls Efficacy Scale (FES), that tries to quantitatively evaluate fear of falling. FES doesn't directly evaluate the degree of fear of falling. Rather, it evaluates the degree of self-efficacy in executing ADLs without falling. High values equate to high confidence with little fear of falling. Hill ${ }^{3)}$ introduced the Modified Falls Efficacy Scale (MFES) adding four items related to outdoor activities. Although many cross-sectional studies have reported correlations between fall-related self-efficacy, balance ability ${ }^{4)}$ and $\mathrm{ADLs}^{5}$, little prospective research ${ }^{6}$ has been done to confirm them.

It is possible that elderly people might be prone to falling when they can't adequately recognize their declining physical abilities. Robinovitch ${ }^{7)}$ evaluated the perception of postural limits of the elderly to investigate the gap between their actual ability and their estimated ability. They measured the gap between the actual reach distance and the estimated reach distance (GAE). The participants' own postural limits were defined as overestimated when the estimated reach distance was further away than the actual reach distance.

A physical therapist should consider recognitive factors such as fall-related self-efficacy and the perception of postural limits in addition to physical abilities. At present, 
Table 1. The reasons why a follow-up survey was impossible

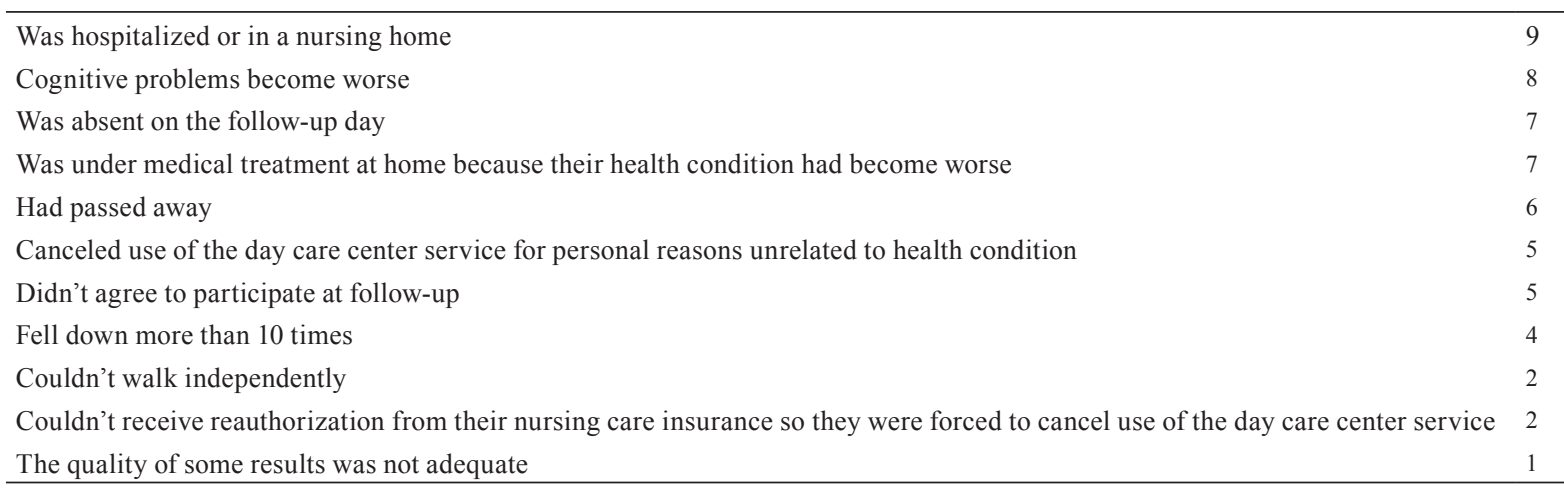

$(n=56)$

there are few prospective studies on whether these recognitive factors influence falls by elderly people.

The purpose of this study was to investigate whether fall-related self-efficacy, perception of postural limits, and general balance of elderly people influence recurrent falls over the period of one year.

\section{SUBJECTS AND METHODS}

At baseline, the participants were 141 elderly cared for at day care centers, who were able to walk independently either without any aids or with some aids such as a $\mathrm{T}$ cane or a walker. All participants scored more than 20 points on the Hasegawa Dementia Scale-revised. Eighty-five participants (mean age: 81.8 years old, range: 66-97, 24 males / 61 females) were available for the follow-up one year later and were targeted for analysis; 56 participants were unavailable at follow-up for the reasons listed in Table 1.

This study was carried out after obtaining the informed consent of all participants.

At baseline and at follow-up, the participants were asked how many times they had fallen in the past year. A fall was defined as an involuntarily event resulting in a knee, hand or some higher body part touching the ground ${ }^{1)}$. We also evaluated participants' fall-related self-efficacy using MFES $^{3)}$ through an interview. All participants were asked how much confidence they had to execute 14 ADLs and outdoor activities without falling. They were scored from 0 points, when the participant didn't have any confidence at all to execute the activity without falling, to 10 points when the participant was completely confident of not falling. A higher value shows a participant has a high fall-related self-efficacy.

We evaluated the recognitive error of postural limits referring to a method described by Robinovitch ${ }^{7)}$. First, the participants estimated their maximum forward reach distance; then, they executed a forward reach. To conduct the test, the participants stood upright with their feet approximately shoulder-width apart, with their arms at their sides, and with their shoulder close to, but not touching a white board. We measured the distance that they reached while bending forward as far as possible without lifting their heels and without falling referring to the Functional Reach Test of Duncan ${ }^{8}$. GAE was defined as the difference between estimated reach distance and actual reach distance, with positive values representing an underestimate, and negative values representing an overestimate.

The Timed Up-and-Go Test (TUG) ${ }^{9)}$ was conducted twice after one practice and the fastest result was recorded.

We assigned whether a participant had fallen more than twice in the year of follow-up to a dependent variable, and with MFES, GAE and TUG at baseline as independent variables, we performed a multiple logistic regression analysis using a forced entry method to explore factors related to recurrent falls. The level of significance was chosen as less than 5\%. PASW Statistics 18 was used for the statistical analysis.

\section{RESULTS}

Twenty-nine participants (34\%) had fallen down in the past year at baseline, and the mean number of falls was 0.81 \pm 1.79 times. Twenty-six participants $(31 \%)$ had fallen in the past year at follow-up, and the mean number of falls was $0.72 \pm 1.55$ times. Fourteen participants $(16 \%)$ had fallen more than twice at follow up. MFES was $116.13 \pm 29.04$ points (Mean $\pm \mathrm{SD}$ ) and GAE was $0.38 \pm 8.39 \mathrm{~cm}$. TUG was $14.99 \pm 6.53$ seconds and FRT was $18.95 \pm 8.54 \mathrm{~cm}$.

Only GAE was determined as a factor related to recurrent falls in the year of follow-up. The result of the Model Chi-square test was significant $(p<0.05)$. The $p$ value of the Hosmer-Lemeshow test was 0.793 . The success rate of discrimination was satisfactory $(83.5 \%)$ (Table 2). There weren't strong correlations among the independent variables so it was judged that multicollinearity didn't exist in this analysis (Table 3 ).

\section{DISCUSSION}

Recurrent falls in one year was prospectively related only to GAE, and didn't relate to general balance. Viccatro and colleagues ${ }^{10)}$ reported that TUG predicted a decline in global health, in addition to an increase in new ADL difficulties and the number of falls. Shumway-Cook and colleagues ${ }^{11)}$ compared TUG values between 15 elderly with a history of 2 or more falls in the previous 6 months and 15 elderly with no history of falls. Their results suggest that TUG could be 
Table 2. The Multiple Logistic Progression Model with exploratory variables

\begin{tabular}{lccc}
\hline Variable & Coefficient $(\beta)$ & Odds Rates & $95 \% \mathrm{CI}$ \\
\hline GAE & $0.08^{*}$ & 1.09 & $1.01-1.17$ \\
Intercept & $-1.81^{* *}$ & & \\
\hline
\end{tabular}

the Model Chi-square test, ${ }^{*} ; \mathrm{p}<0.05,{ }^{* *} ; \mathrm{p}<0.01$. Distinction hitting ratio $83.6 \%$. GAE; the gap between the actual reach distance and the estimated reach distance

Table 3. Correlation coefficients between MFES and GAE and TUG

\begin{tabular}{lcc}
\hline & GAE & TUG \\
\hline MFES & -0.10 & $-0.53^{* *}$ \\
GAE & & 0.13 \\
\hline
\end{tabular}

$(\mathrm{n}=85)$ By Spearman's rank correlation coefficient. ${ }^{* *} \mathrm{p}<0.01$. MFES; the Modified Falls Efficacy Scale. GAE; the gap between the actual reach distance and the estimated reach distance. TUG; the Timed Up-and- Go Test

useful in predicting the probability of falls for elderly people.

We prospectively investigated the factors related to predicting recurrent falls. Viccatro ${ }^{10)}$ didn't assign single falls or recurrent falls to a dependent value and ShumwayCook ${ }^{11)}$ investigated falls retrospectively. The differences in the experimental method of each study may explain why our research arrived at different results.

Robinovitch $^{7)}$ explored whether, when compared to young community-dwelling individuals, elderly participants, either living in nursing homes or being cared for in day care centers, have less accurate perceptions of their postural stability limits. Young participants tended to underestimate their reaching limits, while elderly participants tended to overestimate them. Furthermore, less able reachers tended to overestimate their abilities more. This suggests that elderly people, especially those with impaired postural limits, lack the potential "safety factor" of underestimating their postural limits, as was observed in young participants. Therefore, the elderly may tend to plan movements which create loss of balance. Suzuki and colleagues ${ }^{12)}$ explored factors related to GAE in 263 community-dwelling elderly people. In their result, GAE was related only to age and not to participants' cognitive functions (for example: learning, memory, attention and visuospatial cognition). Suzuki suggested the cognitive characteristic of elderly people who overestimated their physical abilities might be specific to their motor behavior. However these mechanisms have not yet been clarified.

Butler and colleagues ${ }^{13)}$ reported that poor performers were dichotomous, being those who either underestimated or overestimated their postural limits. In addition, fall rates were strongly associated with reach distance but not with reach judgment error. A prior study reported the fall rate of elderly subjects in day care centers as $25.3 \%{ }^{14)}$; in this study, the rate was more than $30 \%$. The standard cut-off value for FRT that is related to a higher risk of falling is $15.3 \mathrm{~cm}^{8)}$.
Also, the cut-off value of TUG for falls is reported to be 13.5 seconds, and is less than 10 seconds for healthy elderly people ${ }^{11)}$. In comparison with these reports, the participants in our study would be considered poor performers, and it is possible that GAE was determined as a factor predicting recurrent falls because of our participants' characteristics.

Okada and colleagues ${ }^{15)}$ retrospectively investigated the relationship between GAE and falls in community-dwelling elderly. They found that GAE was related to recurrent falls. Sugihara and colleagues ${ }^{16)}$ reported it was possible to use this information to predict the risk of falling within three months of the assessment, if GAE was more than $6.5 \mathrm{~cm}$. We found the same to be true even within one year.

If elderly people overestimate their physical abilities, it may lead to recurrent falls. Moreover, our result suggests GAE is a useful assessment index for programs aimed at preventing recurrent falls by elderly people. Some limitations of this study should be noted. We evaluated relatively few subjects and their abilities did not change significantly. One year might be an insufficient time to observe significant change. Also, further research regarding the mechanisms by which elderly people overestimate their abilities is needed.

\section{REFERENCES}

1) Mano Y: The fall in aged people-its cause and its prevention. Tokyo: Ishiyaku, 2002, pp 1-7.

2) Tinetti ME, Powell L: Fear of falling and low self-efficacy: A cause of dependence in elderly persons. J Gerontol, 1993, 48: 35-38. [Medline]

3) Hill KD, Schwarz JA, Kalogeropoulos AJ, et al.: Fear of falling revisited. Arch Phys Med Rehabil, 1996, 77: 1025-1029. [Medline] [CrossRef]

4) Andresen EM, Wolinsky FD, Miller JP, et al.: Cross-sectional and longitudinal risk factors for falls, fear of falling, and falls efficacy in a cohort of middle-aged African Americans. Gerontologist, 2006, 46: 249-257. [Medline] [CrossRef]

5) Kato C, Ida K, Harada A: Influence of high Fall-related self-efficacy on falls due to dissociation with ADL among elderly women in nursing homes. Nippon Ronen Igakkai Zasshi, 2009, 46: 428-435. [Medline] [CrossRef]

6) Mendes de Leon CF, Seeman TE, Baker DI, et al.: Self-efficacy, physical decline, and change in functioning in community-living elders: a prospective study. J Gerontol, 1996, 51: 183-189.

7) Robinovitch SN, Cronin T: Perception of postural limits in elderly nursing home and day care participants. J Gerontol A Biol Sci Med Sci, 1999, 54: B124-B130. [Medline] [CrossRef]

8) Duncan PW, Weiner DK, Chandler J, et al.: Functional reach a new clinical measure of balance. J Gerontol, 1990, 45: M192-M197. [Medline]

9) Podsiadlo D, Richardson S: The timed "Up \& Go": a test of basic functional mobility for frail elderly persons. J Am Geriatr Soc, 1991, 39: 142-148. [Medline]

10) Viccaro LJ, Perera S, Studenski SA: Is timed up and go better than gait speed in predicting health, function, and falls in older adults? J Am Geriatr Soc, 2011, 59: 887-892. [Medline] [CrossRef]

11) Shumway-Cook A, Brauer S, Woollacott M: Predicting the probability for falls in community-dwelling older adults using the Timed Up \& Go Test. Phys Ther, 2000, 80: 896-903. [Medline]

12) Suzuki H, Sakurai R, Fujiwara Y: Does misestimation of physical ability affect decline in physical ability and fall incidence in older adults? Research-Aid Rep, 2011, 26: 49-57.

13) Butler AA, Lord SR, Fitzpatrick RC: Reach distance but not judgment error is associated with falls in older people. J Gerontol A Biol Sci Med Sci, 2011, 66A: 896-903. [Medline] [CrossRef]

14) Suzukawa M, Shimada $H$, Makizako $H$, et al.: Incidence of falls and fractures in disabled elderly people utilizing long-term care insurance. Nippon Ronen Igakkai Zasshi, 2009, 46: 334-340. [Medline] [CrossRef]

15) Okada Y, Takatori K, Nagino K, et al.: Relationship between error in estimated reach distance and falls in community-dwelling elderly people. J Jpn Phys Ther Assoc, 2008, 35: 279-284.

16) Sugihara T, Go T, Mishima $S$, et al.: Elderly people's physical strength awareness and falling. Rigakuryoho Kagaku, 2005, 20: 13-16. [CrossRef] 